\title{
QTc, Tp-e Interval and Tp-e/QTc Ratio in Patients with Hypocalcemia-case Control Study
}

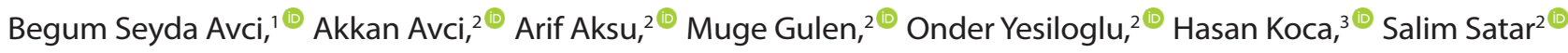 \\ Health Science University, Adana City Research and Training Hospital, Department of Internal Medicine, ${ }^{1}$ Adana - Turkey \\ Health Science University, Adana City Research and Training Hospital, Department of Emergency Medicine, ${ }^{2}$ Adana - Turkey \\ Health Science University, Adana City Research and Training Hospital, Department of Cardiology, ${ }^{3}$ Adana - Turkey
}

\section{Abstract}

Background: To the best of our knowledge, there are studies related to QT and QTc interval in patients with hypocalcemia, but there are no studies evaluating T wave peak and end interval (Tp-e interval), Tp-e/QT and Tp-e/ QTc ratios used to evaluate cardiac arrhythmia risk and ventricular repolarization changes rates.

Objectives: Therefore, we aimed to investigate whether there is a change in Tp-e interval, Tp-e/QT and Tp-e/QTc ratios in patients with hypocalcemia.

Methods: Retrospectively, 29 patients with hypocalcemia in the emergency department were included in the study. Twenty-nine patients with similar age and sex distribution were included in the study as the control group. All patients underwent 12-lead electrocardiography (ECG). In addition to routine measurements, Tp-e interval, Tp-e/QT and Tp-e/QTc ratios were measured on ECG. The study data were grouped as patients with and without hypocalcemia.

Results: The mean age of the patients was $66.24 \pm 4.95$ years. QTc interval, Tp-e interval and Tp-e/QTc values were found to be significantly higher in patients with hypocalcemia ( $\mathrm{p}<0.001$ for each). QTc interval, Tp-e interval and Tp-e/QTc ratio showed a significant negative correlation with calcium levels.

Conclusion: Tp-e interval and Tp-e/QTc ratios are significantly increased in patients with hypocalcemia compared to those without hypocalcemia and this can be used more effectively in the follow-up of cardiac fatal arrhythmias. (Int J Cardiovasc Sci. 2021; [online].ahead print, PP.0-0)

Keywords: Hypocalcemia; QTc; Tp-e interval; Tp-e/QTc ratio.

\section{Introduction}

Hypocalcemia is one of the common electrolyte disorders which can be encountered in the emergency department. It has a wide clinical range from asymptomatic cases to severe life-threatening cases ${ }^{1}$. One of the risk factors leading to prolonged QT interval is hypocalcemia ${ }^{2}$. When cardiac abnormalities related to hypocalcemia are examined, prolongation of QT interval is reported to be the most common ${ }^{3}$. Cases showing that prolonged QT interval associated with hypocalcemia causes fatal ventricular arrhythmias such as Torsades de Pointes are known ${ }^{4,5}$.
There are multiple electrocardiography (ECG) measurements associated with ventricular repolarization and are associated with a risk of ventricular arrhythmia. These measurements are QT and QTc interval, QT and QTc dispersion and T wave peak and end interval (Tpe interval). Among these parameters, QT and QTc are indicative of ventricular depolarization in addition to repolarization. However, Tp-e is more predictive of ventricular repolarization and may be more meaningful especially in the evaluation of repolarization. The obtained Tp-e/QT and Tp-e/QTc ratios are related to ventricular transmural dispersion during repolarization ${ }^{6}$. Increased Tp-e interval shows abnormal spread in ventricular repolarization and is associated with 
increased risk of ventricular arrhythmia ${ }^{7}$. When the literature is reviewed, it is seen that there is no research on Tp-e interval, Tp-e/QT and Tp-e/QTc ratios used in the evaluation of ventricular repolarization in patients with hypocalcemia in the emergency department.

In this study, we aimed to determine whether there was a change in QT, QTc, Tp-e interval, Tp-e/QT and Tp-e/QTc ratios in patients with hypocalcemia in the emergency department compared to patients without hypocalcemia.

\section{Material and Method}

The study was planned as a retrospective case control study. Records of patients who applied to Adana City Research and Training Hospital, Department of Emergency Medicine between January 1, 2019 and August 31, 2019, and whose biochemical tests revealed hypocalcemia were retrospectively examined. Electrocardiography (ECG) recordings obtained from the files of these patients were examined. A total of 29 patients were included in the study. These 29 patients included in the study were defined as the patient group. Biochemical parameters of the patients admitted to the emergency department for various reasons and found to be healthy were examined. ECG recordings of these patients with normocalcemia were obtained. 29 outpatients who were found to be healthy were included in the study as the control group.

Exclusion criteria for all patients included in the study and control group were all medical treatments known to extend or shorten QT and QTc distance, known syncope or sudden cardiac arrest history in the patients or their family, presence of acute or chronic systemic or local infection, being in the pediatric age group ( $<18$ years), inability to perform Tp-e and QTc measurements on ECG, presence of known coronary artery disease (CAD) or diabetes mellitus, one of the major risk factors for CAD, medium-advanced valvular disease, systolic heart failure, electrolyte deficiency, and having the diagnosis of chronic liver disease or chronic renal failure. The study was conducted in accordance with the Helsinki Declaration and approved by the Local Ethics Committee Adana City Research and Training Hospital, Ethics Committee, Meeting Number: 45, Decision No: 630, 04/12/2019.

12-lead ECG and laboratory results of all patients were obtained from the files. From the demographic variables of the patients, age, sex, pulse, blood pressure, oxygen saturation values of all patients were recorded from the archived files. From the routine biochemistry parameters, renal function tests, serum electrolytes, liver function tests were recorded.

\section{2 - Lead Electrocardiographic Evaluation}

Firstly, 12-lead ECG obtained by MAC 2000 ECG Machine (GE medical systems information technologies, Inc., WI, USA) with a sinus rhythm of $25 \mathrm{~mm} / \mathrm{sec}$ and $1 \mathrm{mv} / 10 \mathrm{~mm}$ standard calibration was obtained from the files. All patients were evaluated for the PR interval, from the beginning of the P wave to the beginning of the QRS complex. QRS time was measured from the beginning to the end of the QRS complex. The time from QRS to the point where $\mathrm{T}$ wave returns to the isoelectric line was calculated for the QT time. QTc was calculated using the Bazett Formula $(Q T c=Q T / \sqrt{ } R-R)$. The Tp-e interval was defined as the time from the peak of the $\mathrm{T}$ wave to the point where the $\mathrm{T}$ wave interconnected with the isoelectric line. Measurements were made primarily from V5. If V5 was unsuitable for measurement (amplitude $<1.5 \mathrm{~mm}$ ), measurements were taken from $\mathrm{V} 4$ or $\mathrm{V}^{8}$. Tp-e/ QTc ratio was calculated based on these measurements. All ECG examinations in sinus rhythm were evaluated by two cardiologist with at least 5 years of experience in electrophysiology and $\geq 2000$ arrhythmia patients annually, while unaware of the clinical status of the patient.

\section{Statistical Analysis}

All analyzes were performed using SPSS 22.0 (Chicago, IL, USA) statistical software package. Kolmogorov-Smirnov test was used to determine whether the distribution of continuous variables was normal. Continuous variables in the group data were expressed as mean \pm standard deviation. Categorical variables were expressed as numbers and percentages. Continuous variables that showed normal distribution was compared using the Student $t$ test, whereas the Mann-Whitney $U$ test is used to compare differences between two independent groups when the dependent variable is either ordinal or continuous, but not normally distributed. Student $t$ test used was paired. Chi-square $\left(\chi^{2}\right)$ test was used to compare categorical variables. The kappa coefficient was used to examine the interobserver variability of all ECG measurements. Both Pearson's and Spearman's correlation analysis was used to determine the presence of a relationship between countable parameters. Linear regression analysis of parameters showing statistically significant correlation was done and beta values were obtained. All the assumptions necessary to use the linear regression analysis were verified. Statistical significance level was accepted as $\mathrm{p}<0.001$. 


\section{Results}

The study data were divided into two groups as patient and control groups. Electrocardiographic measurements were taken successfully from all patients included in the study.

When demographic data were compared according to the study groups, age and sex were found to be similar between the groups. Laboratory parameters were similar between the two groups (Table 1).

When ventricular repolarization parameters were examined according to the study groups, QTc interval, Tp-e interval and Tp-e/QTc values were found to be significantly higher in patients with hypocalcemia (Table 2).

Table 3 shows the correlation of QTc, Tp-e-interval and Tp-e/QTc measurements with the calcium and ionized calcium parameters. All measurements negatively correlated with calcium and ionized calcium levels (Table 3). Linear regression analysis was performed with calcium being significantly related to QTc, Tp-e-interval
Tp-e/QTc measurements (Table 4). In linear regression analyses, QTc, Tp-e-interval and Tp-e/QTc ratio were found to be independently associated with calcium level. In Scatterplot analyses of calcium levels with QTc interval, Tp-e interval and Tp-e/QTc ratios, $\mathrm{R}^{2}$ linear values were $0.656,0.818$ and 0.785 , respectively (Figure 1, Figure 2 and Figure 3).

\section{Discussion}

The main finding of our study was that the patients with hypocalcemia had significantly higher QTc, Tp-e interval and Tp-e/QTc ratios than those without hypocalcemia. To the best of our knowledge, this finding is the first study in the literature to show an increase in Tp-e interval and Tp-e/QTc ratios among the ventricular repolarization parameters in patients with hypocalcemia. Our study also supported previous studies showing QT and QTc prolongation in patients with hypocalcemia.

Ventricular myocardium depolarization occurs from the endocardial region towards the epicardial region.

Table 1 - Comparison of Demographic and Laboratory Findings between Hypocalcemia and Control Group

\begin{tabular}{|c|c|c|c|}
\hline & $\begin{array}{l}\text { Patients with Hypocalcemia } \\
\qquad \mathrm{n}=29\end{array}$ & $\begin{array}{l}\text { Patients without } \\
\text { Hypocalcemia } \\
\text { n=29 }\end{array}$ & $\mathrm{p}$ \\
\hline Age (years) & $66.24 \pm 4.95$ & $64.07 \pm 4.52$ & 0.087 \\
\hline Systolic blood pressure (mmHg) & $114.83 \pm 12.14$ & $118.28 \pm 11.67$ & 0.275 \\
\hline Diastolic blood pressure (mmHg) & $70.69 \pm 9.23$ & $72.07 \pm 8.19$ & 0.550 \\
\hline Heart rate (pulse/minute) & $118.76 \pm 12.40$ & $82.76 \pm 10.79$ & $<0.001$ \\
\hline Urea (mg/dL) & $34.38 \pm 8.96$ & $37.00 \pm 7.02$ & 0.221 \\
\hline Creatinine (mg/dL) & $0.75 \pm 0.24$ & $0.78 \pm 0.21$ & 0.650 \\
\hline Sodium (mEq/L) & $139.17 \pm 2.67$ & $139.24 \pm 3.28$ & 0.930 \\
\hline Potassium (mEq/L) & $4.15 \pm 0.51$ & $4.23 \pm 0.37$ & 0.462 \\
\hline Glucose (mg/dL) & $103.76 \pm 13.24$ & $108.24 \pm 12.03$ & 0.183 \\
\hline $\operatorname{ALT}(\mathrm{u} / \mathrm{L})^{*}$ & $31.21 \pm 12.50$ & $18.97 \pm 14.56$ & 0.009 \\
\hline $\operatorname{AST}(\mathrm{u} / \mathrm{L})^{* *}$ & $24.10 \pm 8.26$ & $25.97 \pm 12.27$ & 0.501 \\
\hline Albumine (g/L) & $40.14 \pm 3.01$ & $42.00 \pm 4.83$ & 0.085 \\
\hline Calcium (mg/dL) & $6.90 \pm 0.65$ & $9.47 \pm 0.35$ & $<0.001$ \\
\hline Ionized Calcium (mmol/L) & $0.98 \pm 0.11$ & $1.07 \pm 0.06$ & $<0.001$ \\
\hline
\end{tabular}


Table 2 - Comparison of Ventricular Repolarization Parameters between Hypocalcemia and Control Group

\begin{tabular}{|c|c|c|c|}
\hline & $\begin{array}{l}\text { Patients with Hypocalcemia } \\
\qquad n=29\end{array}$ & $\begin{array}{l}\text { Patients without } \\
\text { Hypocalcemia } \\
\text { n=29 }\end{array}$ & p \\
\hline QTc interval time (ms) & $476.59 \pm 22.51$ & $432.62 \pm 9.28$ & $<0.001$ \\
\hline Tp-e interval time (msn) & $128.38 \pm 15.34$ & $77.38 \pm 8.38$ & $<0.001$ \\
\hline Tp-e/QTc Ratio & $26.91 \pm 2.72$ & $17.87 \pm 1.81$ & $<0.001$ \\
\hline
\end{tabular}

Table 3 - Correlation of QTc, Tp-e Interval and Tp-e/QTc Raito with Calcium and Ionized Calcium

\begin{tabular}{lccccccc}
\hline & \multicolumn{3}{c}{ QTc } & & Tp-e-interval & & Tp-e/QTc Ratio \\
\cline { 2 - 8 } & $\mathbf{r}$ & $\mathbf{p}$ & $\mathbf{r}$ & $\mathbf{p}$ & $\mathbf{r}$ & $\mathbf{p}$ \\
\hline Calcium (mg/dL) & -0.810 & $<0.001$ & -0.904 & $<0.001$ & -0.886 & $<0.001$ \\
\hline Ionized Calcium (mmol/L) & -0.508 & $<0.001$ & -0.499 & $<0.001$ & -0.459 & $<0.001$ \\
\hline
\end{tabular}

Table 4 - A Linear Regression Analysis for Calcium Significantly Correlated with QTc, Tp-e Interval and Tp-e/QTc Raito

\begin{tabular}{|c|c|c|c|c|c|c|}
\hline & \multicolumn{2}{|c|}{ QTc } & \multicolumn{2}{|c|}{ Tp-e-Interval } & \multicolumn{2}{|c|}{ Tp-e/QTc Ratio } \\
\hline & $\beta$ & p & $\beta$ & p & $\beta$ & p \\
\hline Calcium & -0.309 & $<0.001$ & -0.807 & $<0.001$ & -0.647 & $<0.001$ \\
\hline
\end{tabular}

After depolarization, ventricular repolarization occurs. During ventricular repolarization, there is dispersion between the endocardial and epicardial region. The intervals between the $\mathrm{T}$ wave peak and the end distance are called the Tp-e interval, which is associated with transmural ventricular repolarization ${ }^{6,9}$. Tp-interval and its ratio to the QT interval have been shown to be associated with an arrhythmic clinical status in many cardiac pathological conditions and also poses a high risk for sudden cardiac death ${ }^{5,10-12}$. Increased Tp-e interval and Tp-e/QTc ratio are associated with arrhythmia and sudden cardiac death, dispersion in the epicardial and endocardial regions of the ventricular myocardium between the epicardial and endocardial regions causes slow conduction in these two anatomic regions and this is thought to create an increase in re-entry, which is the most common cause of arrhythmias.
Hypocalcemia characteristically results in prolongation of the ST segment and a long QTc interval ${ }^{4,13}$. Although there are studies evaluating QT and QTc interval, which are among the ventricular repolarization parameters, in patients with hypocalcemia, there are no studies investigating the Tp-e interval, Tp-e/QT and Tp-e/QTc ratio $^{2}$. Two studies show that prolongation of QTc is an independent risk factor for hypocalcemia ${ }^{14,15}$. In our study, the obtained QTc intervals were increased in accordance with previous literature. In addition, the increase in Tp-e-interval and Tp-e/QTc ratios were markedly more significant in patients with hypocalcemia when compared to the control group. Increased ventricular repolarization parameters such as QT and QTc duration, QTc dispersion, Tp-e interval, Tp-e/QTc have been shown 


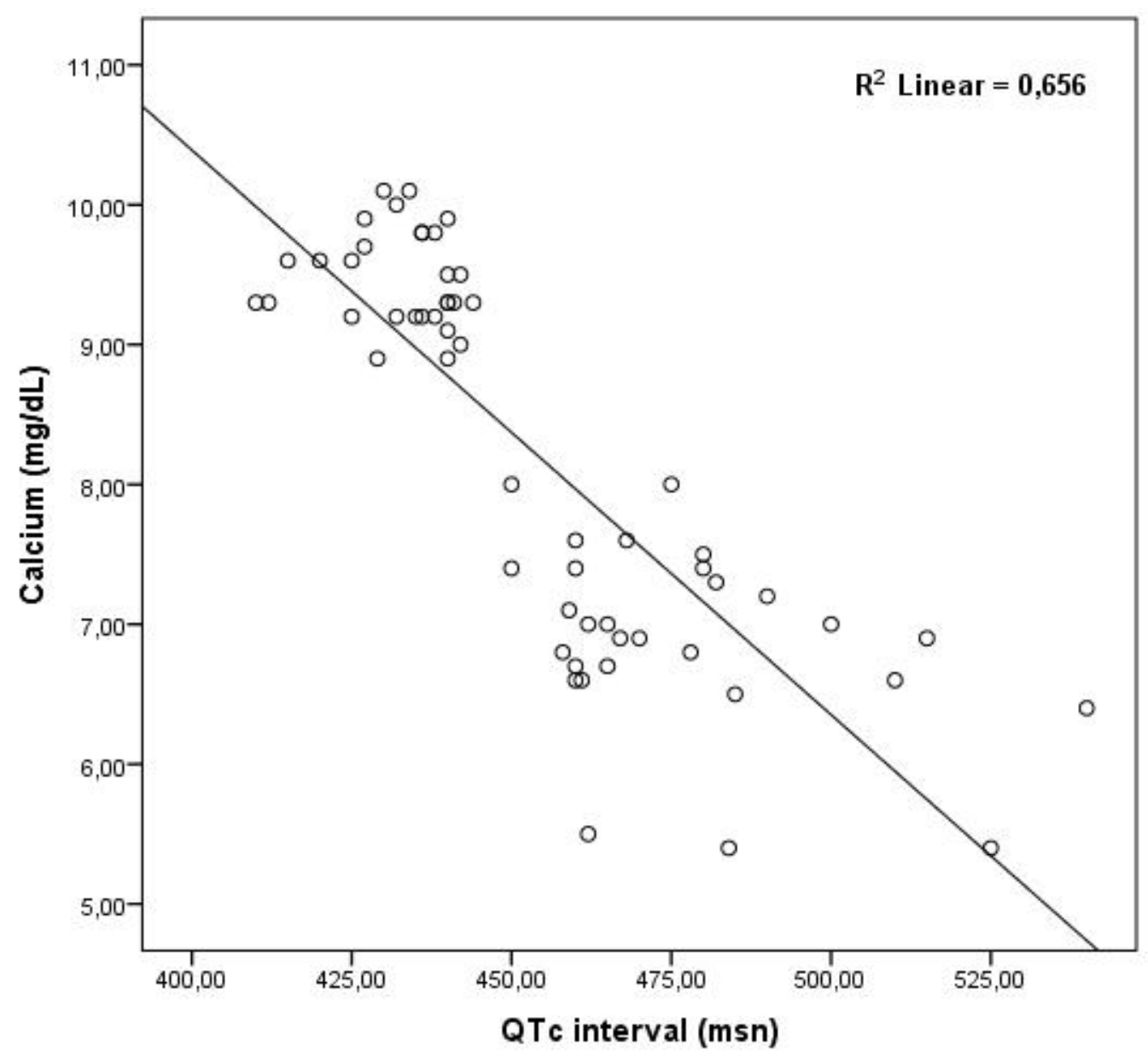

Figure 1 - Analysis of Scatterplot for the relationship between Calcium and QTc interval.

to be risk factors for ventricular arrhythmia and death ${ }^{6,7}$. Increased QTc may cause life-threatening torsades de pointes ${ }^{16}$. In our study, it was shown for the first time that Tp-e interval and Tp-e/QTc increased in hypocalcemia patients. Hypocalcemia may appear as Torsades de pointes due to prolonged QTc interval. This is due to the prolongation of the plateau phase of cardiac action potential depending on the calcium level ${ }^{17,18}$. Tp-e and Tp-e/QTc parameters, as well as prolongation of QTc distance due to hypocalcemia, may be a predictor of ventricular dispersion and repolarization. Therefore, early diagnosis and close monitoring of ventricular repolarization changes in hypocalcemia patients should be performed. According to the data obtained in our study, we think that in addition to QT and QTc intervals, evaluation of Tp-e and Tp-e/QTc ratio may be more useful in the evaluation of ventricular repolarization.

\section{Limitations of Study}

There are some important limitations in our study. One of them is the retrospective design of the study, and also the number of patients included in the study. In our study, the number of patients was limited to 29. Prospective studies involving more patients may provide more meaningful information.

\section{Conclusion}

Tp-e interval and Tp-e/QTc ratios were significantly increased in patients with hypocalcemia. In addition to QT and QTc evaluation during routine ECG evaluation in patients with hypocalcemia detected in emergency departments, it is important to keep Tp-e-interval and Tp-e/QTc ratios in mind, which are other ventricular repolarization parameters, and that 


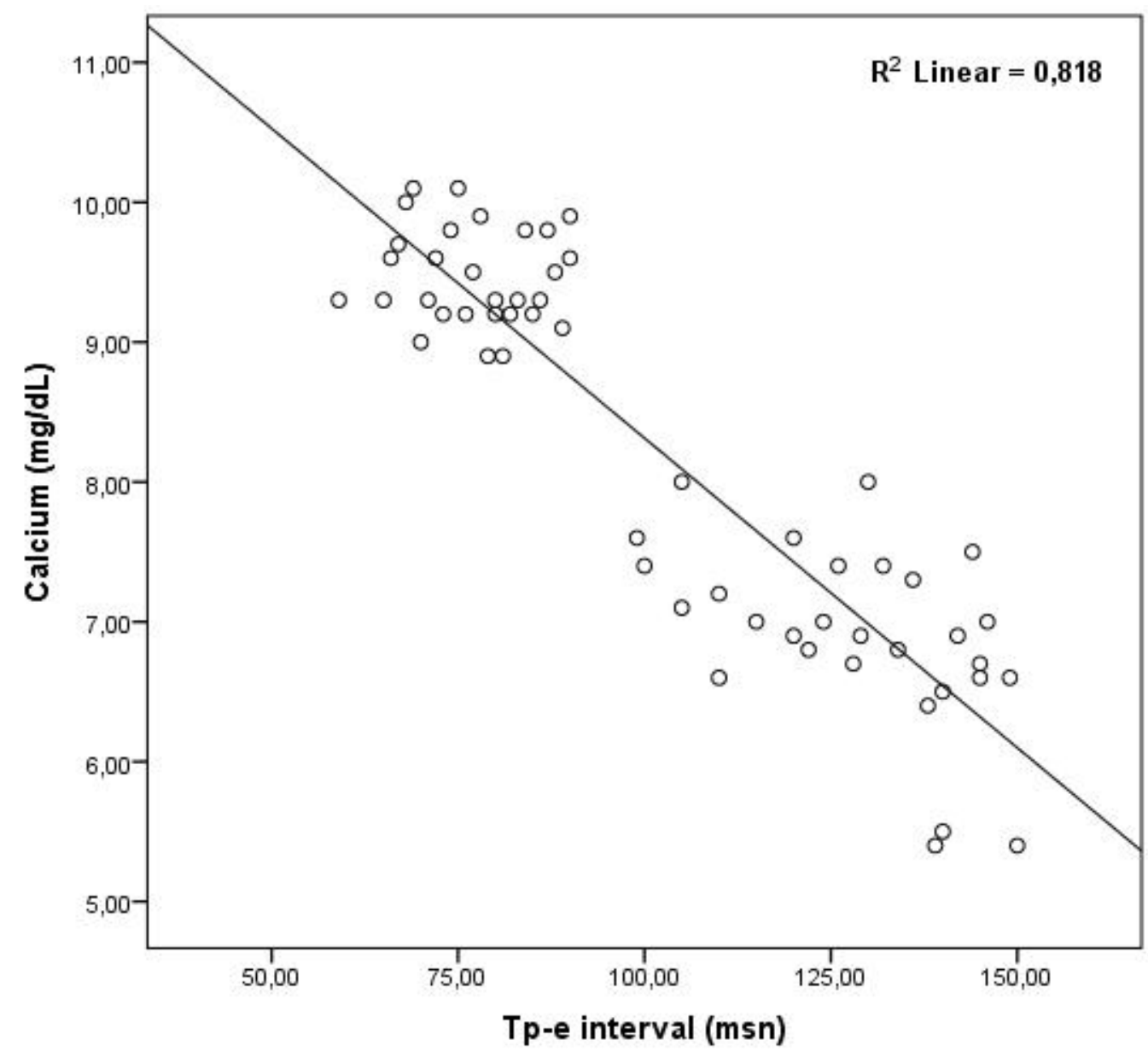

Figure 2 - Analysis of Scatterplot for the relationship between Calcium and Tp-e interval.

cardiac lethal arrhythmias may be seen more frequently in cases where these values are higher. However, as this information is shown for the first time in our study, studies involving new and more patients with hypocalcemia should be performed.

\section{Author Contributions}

Conception and design of the research: Avci BS, Avci A. Acquisition of data: Aksu A, Koca H. Statistical analysis: Gulen M, Satar S. Writing of the manuscript: Avci BS. Critical revision of the manuscript for intellectual content: Avci BS, Avci A, Aksu A, Koca H, Gulen M, Satar S.

\section{Potential Conflict of Interest}

No potential conflict of interest relevant to this article was reported.

\section{Sources of Funding}

There were no external funding sources for this study.

\section{Study Association}

This study is not associated with any thesis or dissertation work.

\section{Ethics Approval and Consent to Participate}

This study was approved by the Ethics Committee of the Adana City Research and Training Hospital under the protocol number Meeting Number: 45, Decision No: 630, 04/12/2019. All the procedures in this study were in accordance with the 1975 Helsinki Declaration, updated in 2013. Informed consent was obtained from all participants included in the study. 


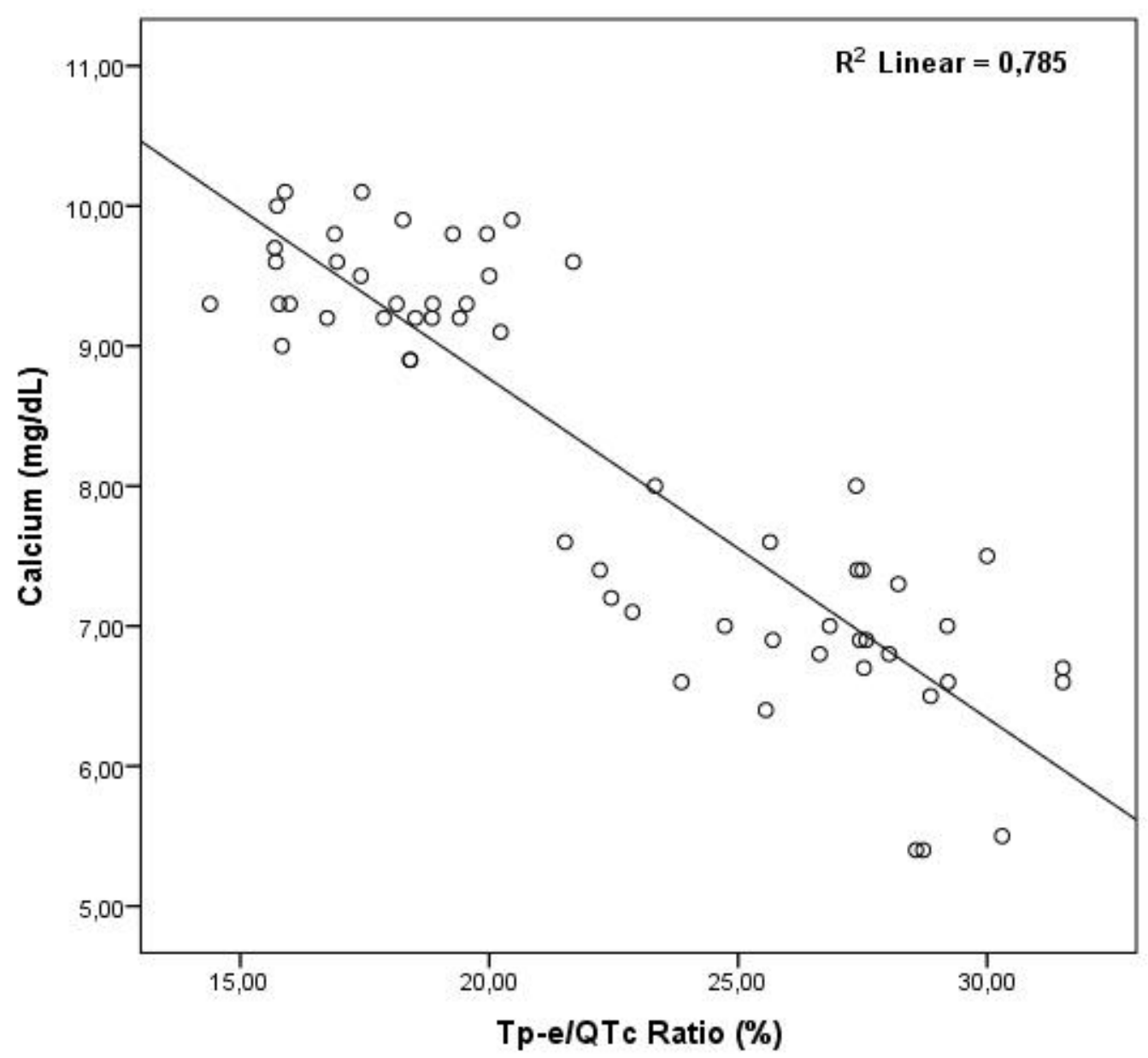

Figure 3 - Analysis of Scatterplot for the relationship between Calcium and Tp-e/QTc ratio.

\section{References}

1 Rossi GP, Bernini G, Caliumi C, Desideri G, Fabris B, Ferri C, et al. A prospective study of the prevalence of primary aldosteronism in 1,125 hypertensive patients. J Am Coll Cardiol. 2006;48(11):2293-300.

2. Charlotte PM, Marieke PK, Florine AB, Patricia ML, Aaf FM, Ruud TM, et al. Risk factors for QTc interval prolongation. Eur J Clin Pharmacol. 2018; 74(2):183-91.

3. Newman DB, Fidahussein SS, Kashiwagi DT, Kennel KA, Kashani $\mathrm{KB}$, Wang Z, et al. Reversible cardiac dysfunction associated with hypocalcemia: a systematic review and meta-analysis of individual patient data. Heart Fail Rev. 2014 Mar;19(2):199-205.

4. Shibba TC, Shivani M, Sandeep C, Manikant S, Naved A, Bishav M, et al. Hypocalcemia Presenting as Life Threatening Torsades de Pointes with Prolongation of QTc Interval. Ind J Clin Biochem. 2018;33(2):235-8.

5. Filip MS, Grzgorz K, Anna EP, Bartosz P, Krzyszof JF. Long QT interval in a patient after out-of-hospital cardiac arrest with hypocalcaemia, undergoing therapeutic hypothermia. Am J Emerg Med. 2013 Dec;31(12):1722.e1-3.

6. Kongstad O, Xia Y, Liang Y, Hertervig E, Ljungström E, Olsson B, et al. Epicardial and endocardial dispersion of ventricular repolarization. A study of monophasic action potential mapping in healthy pigs. Scand Cardiovasc J. 2005; 39(6):342-7.
7. Porthan K, Viitasalo M, Toivonen L, Havulinna AS, Jula A, Tikkanen JT, et al. Predictive Value of Electrocardiographic TWave Morphology Parameters and T-Wave Peak to T-Wave End Interval for Sudden Cardiac Death in the General Population. Circ Arrhythm Electrophysiol. 2013 Aug;6(4):690-6.

8. Chua KC, Rusinaru C, Reinier K, Uy-Evanado A, Chugh H, Gunson $\mathrm{K}$, et al. Tpeak-to-Tend interval corrected for heart rate: A more precise measure of increased sudden death risk?. Heart rhythm. 2016; 13(11):2181-5.

9. Xia Y, Liang $Y$, Kongstad O, Holm M, Olsson B, Yuan S. Tpeak-Tend interval as an index of global dispersion of ventricular repolarization evaluations using monophasic action potential mapping of the epi and endocardium in swine. J Interv Card Electrophysiol. 2005;14(2):79-87.

10. Gupta P, Patel C, Patel H, Narayanaswamy S, Malhotra B, Green JT, et al. T(p-e)/QT ratio as an index of arrhythmogenesis. J Electrocardiol. 2008;41(6):567-74.

11. Erikssen G, Liestol K, Gullestad L, Haugaa KH, Bendz B, Amlie JP. The terminal part of the QT interval ( $\mathrm{T}$ peak to $\mathrm{T}$ end): a predictor of mortality after acute myocardial infarction. Ann Noninvasive Electrocardiol. 2012;17(2):85-94. 
12. Smetana P, Schmidt A, Zabel M, Hnatkova K, Franz M, Huber K, et al. Assessment of repolarization heterogeneity for prediction of mortality in cardiovascular disease: peak to the end of the T wave interval and nondipolar repolarization components. J Electrocardiol. 2011:44(3):301-8.

13. Allison BR, Lee B, Gaurav A. Electrolyte screening in the evaluation of prolonged QTc interval. Cardiol Young. 2015; 25(2):398-9.

14. Benoit S, Mendelsohn A, Nourjah P, Staffa J, Graham D. Risk factors for prolonged QTc among US adults: Third National Health and Nutrition Examination Survey. Eur J Cardiovasc Prev Rehabil 12(4):363-8.

15. Sohaib SM, Papacosta O, Morris R, Macfarlane P, Whincup P. Length of the QT interval: determinants and prognostic implications in a population-based prospective study of older men. J Electrocardiol. 41(6):704-10.
16. Sade E, Oto A, Oto A, Oner Z, Daver A, Onalan O, et al. Adrenal adenoma presenting with torsade de pointes: a case report. Angiology. 2002;53(4):471-4.

17. Eryol NK, Colak R, Ozdogru I, Tanriverdi F, Unal S, Topsakal R, et al. Effects of calcium treatment on QT interval and QT dispersion in hypocalcemia. Am J Cardiol. 2003;91(6):750-2.

18. Bronsky D, Dubin A, Waldstein SS, Kushner DS. Calcium and the electrocardiogram: I. The electrocardiographic manifestations of hypoparathyroidism. Am J Cardiol. 1961;7(6):823-32. 